Erica Ell 1

Luiz Antonio Bastos Camacho 2 Dora Chor 2

\section{Perfil antropométrico de funcionários de banco estatal no Estado do Rio de J aneiro/Brasil: I - índice de massa corporal e fatores sócio-demográficos}

\author{
Anthropometric profile of employees \\ from a government bank in the State \\ of Rio de J aneiro, Brazil - I: body mass \\ index and sociodemographic factors
}

1 Departamento de Medicina Social e Preventiva da Universidade Gama Filho. Rua Manoel Vitorino 625, Rio de Janeiro, RJ 20740-280, Brasil.

2 Departamento de Epidemi ol ogia e Métodos Quantitativos em Saúde Pública da Escola

Nacional de Saúde Pública. Rua Leopoldo Bulhões 1480, Rio de Janeiro, RJ 21041-210, Brasil.
Abstract This study first describes the anthropometric profile of employees at Banco do Brasil and then compares these results to those of the Brazilian National Health and Nutritional Survey, PNSN/1989, for the Southeastern region of the country. It is a cross-sectional study with a sample comprising 647 bank clerks. Weight and hei ght informed by respondents were used to compute the body mass index (BMI). Among bank clerks, $8.8 \%$ were underweight, $27.8 \%$ were overweight and $6.4 \%$ were obese. Overweight increased with age and was about 3 times greater in men than in women. Among social and demographic variables, sex, age, and school ing best explained BMI variance. For men, proportion of overweight was higher in bank clerks than in the PNSN population, while for women, PNSN showed higher frequencies of overweight as compared to female bank clerks. High prevalence of overweight in the general population, as well as in specific groups like bank clerks, highlights the urgent need for definition and development of prevention and control policies capable of impacting risk factors and ultimately leading to a decrease in social cost due to mortality from chronic diseases.

Key words Obesity; Nutritional Status; Nutritional Assessment; Epidemiology

Resumo Estudo seccional que objetivou descrever o perfil antropométrico de funcionários do Banco do Brasil e compará-lo aos resultados da Pesquisa Nacional Sobre Saúde e Nutrição PNSN/1989 para a regi ão Sudeste. Utilizou-se uma amostra de 647 bancári os. Entre os funci onários, $8,8 \%$ apresentaram baixo peso, $27,8 \%$ sobrepeso e $6,4 \%$ obesi dade. O excesso de peso aumentou com a idade efoi aproximadamente 3 vezes maior em homens do queem mulheres. Dentre as variáveis sócio-demográficas, o sexo, a idade e a escol ari dadeforam as que mel hor explicaram a variação do IMC. Homens da população Su deste tiveram proporções mais el evadas de baixo peso do que os do Banco do Brasil. Os bancári os homens apresentaram freqüências maiores de sobrepeso comparados aos da PNSN. Para as mul heres, freqüências mais el evadas de sobrepeso e obesidade foram estimadas pela PNSN. As al tas prevalências de excesso de peso mostram a necessidade da implementação de medidas de prevenção e controle, contribuindo para a redução destes índi ces, bem como para a redução de gastos sociais devi dos a morbi-mortali dade por doenças crônicas.

Palavras-chave Obesidade; Estado Nutricional; Avaliação Nutricional; Epidemiologia 


\section{Introdução}

A sociedade moderna tem vivenciado, nas últimas décadas, um dinâmico e complexo processo de mudanças nos padrões alimentares e nutricionais, nos perfis demográfico, sócio-econômico e epidemiológico. Tal processo vem acontecendo de maneira diferenciada em países, regiões e grupos sociais diversos (OMS, 1990; Popkin, 1994). Essas transformações vêm acarretando intensas modificações no quadro de morbi-mortalidade. Doenças infecciosas e parasitárias, estreitamente associadas ao estado nutricional, condições de moradia e saneamento básico estão sendo substituídas pelas doenças crônicas, entre as quais destacam-se as doenças cardiovasculares, por representarem a primeira causa de óbito na maioria dos países ocidentais. Também no Brasil, elas determinam um terço de todas as mortes (Prata, 1992).

Em muitos países desenvolvidos, o controle de fatores considerados de risco para doenças crônicas, tais como hipertensão arterial, hipercolesterolemia, tabagismo, dieta rica em gordura e obesidade, entre outros, vem contribuindo para a prevenção e redução de mortes prematuras, principalmente aquelas causadas por doenças cardiovasculares (OMS, 1990). No Brasil, apesar de escassos, os estudos existentes mostram que os fatores de risco para doenças cardiovasculares, câncer e diabetes têm dimensões e importância similares às observadas nos países desenvolvidos (Lessa et al., 1996).

Com relação ao estado nutricional de populações adultas, tanto em países desenvolvidos como em desenvolvimento, a prevalência de sobrepeso e obesidade vêm aumentando. Nos Estados Unidos, segundo Rippe (1996), dados coletados no NHANES III (National Health and Nutrition Examination Survey) evidenciaram incremento de $8 \%$ na prevalência de sobrepeso entre 1980 e 1990, e um terço da população com mais de 20 anos de idade apresenta peso igual ou superior a $20 \%$ do desejado. Wolf \& Colditz (1996) estimaram que, dado à extrema relevância da associação do sobrepeso/ obesidade com o acréscimo da incidência de doenças cardiovasculares, diabetes mellitus, hipercolesterolemia e algumas formas de câncer, aproximadamente $6,8 \%$ do gasto com estas doenças poderiam ter sido evitados nos Estados Unidos, em 1990, caso se tivesse atuado em prol da prevenção e redução do sobrepeso/ obesidade.

No Brasil, a comparação entre o Estudo Nacional de Despesa Familiar (Endef)/ 1974 e a Pesquisa Nacional sobre Saúde e Nutrição (PNSN)/ 1989, realizadas pela Fundação Instituto Brasileiro de Geografia e Estatística (FIBGE), permite observar que, no período compreendido entre os dois estudos, a prevalência de baixo peso diminuiu em cerca de $36 \%$, tanto em homens como, em mulheres, enquanto o excesso de peso tornou-se duas vezes maior, sendo este acréscimo maior entre os homens do que entre as mulheres (Coitinho et al., 1991).

Comparando-se a prevalência de sobrepeso/ obesidade encontrada na PNSN, com a dos ingleses (1980), canadenses (1981) e americanos (1976-1980), verificou-se uma prevalência geral muito mais baixa entre os homens brasileiros. Entretanto, quando a análise restringiuse àqueles com renda familiar duas vezes maior do que a média brasileira, foram estimadas proporções semelhantes àquelas apresentadas por aqueles países. Em relação às mulheres, tanto a prevalência geral como aquela restrita ao estrato de renda mais elevada foram semelhantes à encontrada entre as americanas, cuja freqüência de sobrepeso/ obesidade é uma das mais altas do mundo (Sichieri et al., 1994).

Outros estudos nacionais estimaram a prevalência de obesidade em populações específicas, e estas variaram entre 18,0\% e 40,1\% (IMC $\geq 27 \mathrm{~kg} / \mathrm{m}^{2}$ ) para ambos os sexos (Rouquayrol et al., 1987; Rego et al.,1990; Lolio \& Latorre 1991; Bloch et al., 1994). Duncan et al. (1993) encontraram, em Porto Alegre, 15,0\% para homens (IMC $\geq 27,8 \mathrm{~kg} / \mathrm{m}^{2}$ ) e $24,0 \%$ para mulheres $\left(I M C \geq 27,3 \mathrm{~kg} / \mathrm{m}^{2}\right)$.

Com o objetivo de avaliar o estado nutricional de populações ou de grupos populacionais, os estudos vêm utilizando largamente dados antropométricos que, conforme Gibson (1990), além de servirem de instrumento de avaliação nutricional, são úteis também para realizar comparações temporais em um mesmo grupo ou entre populações. Os dados analisados nesta pesquisa compõem estudo epidemiológico realizado em 1994, que avaliou funcionários do Banco do Brasil do Estado do Rio de Janeiro quanto às suas condições de saúde, bem como no que diz respeito à utilização de serviços de saúde. Dessa forma, buscou-se traçar o perfil de morbidade e de fatores de risco, tendo em vista que, nas últimas décadas, as doenças cardiovasculares representaram a primeira causa de morte entre os funcionários do sexo masculino (Beltrão et al., 1996). Os resultados aqui apresentados referem-se aos bancários que atuavam nos Cesecs (Centros de Processamento de Serviços e Comunicações). Segundo Griep (1996), no período do levantamento dos dados, 
eram desenvolvidas nos Cesecs atividades relacionadas ao sistema de informações da rede bancária, tais como serviços gráficos e de impressão, compensação de cheques e confecção de cartões magnéticos.

Cabe notar que outros estudos que compararam a morbidade referida e a prevalência de fatores de risco dos funcionários dos Cesecs com aqueles lotados em agências ou na Direção Geral, não encontraram diferenças significativas (Chor, 1997).

Nesta investigação, os principais objetivos são: descrever o perfil antropométrico dos bancários dos Cesecs, verificar sua associação com características sócio-demográficas e compará-lo aos resultados da PNSN para a região Sudeste.

\section{Metodologia}

A população de estudo foi constituída por funcionários do Banco do Brasil ativos do Estado do Rio de Janeiro que atuavam nos 11 (onze) Cesecs (Centros de Processamento de Serviços e Comunicações).

A amostra foi do tipo aleatória simples, com precisão esperada das estimativas estabelecida na ordem de $4 \%$ e nível de significância de $95 \%$.

Os dados foram obtidos através de questionário auto-respondido, aplicado no local de trabalho, durante o horário de expediente, com ajuda de uma equipe de aplicadores treinados. O questionário abordou, entre outros temas, a situação sócio-demográfica, perfil funcional e perfil econômico. A renda familiar (Reais) e o número de membros da família originaram a variável renda familiar per capita mensal, categorizada em quartis. O índice de massa corporal (IMC) foi construído considerando-se o quociente entre o peso em quilogramas ea estatura (em metros) elevados ao quadrado. As classificações adotadas para o IMC foram: 1) a proposta pela Organização Mundial de Saúde OMS, (1986) que estabelece como limites de corte para o IMC $<20 \mathrm{~kg} / \mathrm{m}^{2}$ (baixo peso), IMC entre $20,0-24,9 \mathrm{~kg} / \mathrm{m}^{2}$ (normal), IMC entre 25,0$29,9 \mathrm{~kg} / \mathrm{m}^{2}$ (sobrepeso) e IMC $\geq 30 \mathrm{~kg} / \mathrm{m}^{2}$ (obesidade); 2) a classificação proposta pela OMS (1995), que estabelece IMC $<18,5 \mathrm{~kg} / \mathrm{m}^{2}$ (baixo peso), IMC entre $18,5-24,5 \mathrm{~kg} / \mathrm{m}^{2}$ (normal), IMC entre $25,0-29,9 \mathrm{~kg} / \mathrm{m}^{2}$ (sobrepeso grau I), IMC entre 30,0-40,0 kg/ m² (sobrepeso grau II) e IMC $\geq 40,0 \mathrm{~kg} / \mathrm{m}^{2}$ (sobrepeso grau III).

O banco de dados foi construído com o programa Dbase III (Ashton-Tate, 1988), e para a análise estatística utilizou-se o software SPSS for Windows - Statistical Package for Social Sciences - versão 6.2 (Norusis, 1992). A significância estatística das diferenças entre proporções foi avaliada através do teste do $\chi 2$ (quiquadrado) e as diferenças entre médias foram analisadas uilizando-se o teste t-Student. Foram construídos intervalos de $95 \%$ de confiança (IC - 95\%) e utilizou-se a técnica de regressão linear para modelar a inter-relação da variável dependente (IMC) com as independentes. Para selecionar as variáveis e a ordem de entrada de cada uma no modelo, verificou-sea correlação entre o IMC e cada uma das variáveis preditoras. O critério adotado para a permanência da variável no modelo foi a probabilidade associada ao teste $\mathrm{F}$ com valor em torno de 0,05 (Kahn \& Sempos, 1989).

A distribuição percentual do I MC dos funcionários do Banco do Brasil foi comparada com aquela apresentada na PNSN para a região Sudeste (Coitinho et al., 1991), por sexo e grupos etários.

\section{Resultados}

Dentre os funcionários sorteados nos Cesecs, apenas 3,6\% recusaram-se a participar da pesquisa. A amostra final foi composta por 358 homens (55,3\%) e 289 mulheres (44,7\%). A idade variou entre 22 e 59 anos, sendo a média das mulheres (39,1 anos) significativamente maior ( $p \varangle 0,001$ ) que a dos homens (36,9 anos).

Tanto homens, como mulheres, eram, em sua mai oria, casados (61,4\%). Entre os homens havia maior proporção de solteiros do que entre as mulheres, enquanto o inverso ocorreu para a categoria divorciados/ separados e viúvos (Tabela 1).

A média salarial foi maior para os homens $(\mathrm{R} \$ 1039,5)$ do que para as mulheres $(\mathrm{R} \$ 912,5)$ $(p \varangle 0,001)$. Entretanto, a renda familiar per capita média das mulheres $(\mathrm{R} \$ 657,1)$ foi maior do que a dos homens ( $R \$ 586,5)$, embora sem diferença estatisticamente significativa ( $p=$ 0,08 ). De forma coerente, a proporção de homens no quartil inferior de renda familiar mensal per capita foi inferior à das mulheres (Tabela 1).

Quanto à escolaridade, grande parte dos funcionários chegaram a ingressar na universidade, e maior proporção de mulheres apresentaram nível superior completo $(66,8 \%$; IC $=$ $61,9-71,6)$ comparadas aos homens $(47,2 \%$; IC =42,5-51,9) (Tabela 1).

As medianas do peso para homens e muIheres foram respectivamente $76,5 \mathrm{~kg}$ e $60 \mathrm{~kg}$, sistematicamente maiores nos homens em to- 
Tabela 1

Características sócio-demográficas dos funcionários do Banco do Brasil, por sexo - 1994/RJ.

\begin{tabular}{|c|c|c|c|c|}
\hline & \multicolumn{2}{|c|}{ Homens } & \multicolumn{2}{|c|}{ Mulheres } \\
\hline & $\mathrm{n}$ & $\%$ & $\mathrm{n}$ & $\%$ \\
\hline \multicolumn{5}{|l|}{ Situação conjugal (\%)* } \\
\hline Solteiros & 106 & 29,6 & 57 & 19,7 \\
\hline Casados & 216 & 60,3 & 181 & 62,6 \\
\hline Divorciados/Separados/Viúvos & 36 & 10,1 & 51 & 17,6 \\
\hline \multicolumn{5}{|l|}{ Ativividade funcional (\%) } \\
\hline Administrativo & 346 & 96,6 & 280 & 97,6 \\
\hline Outros quadros & 12 & 3,4 & 07 & 2,4 \\
\hline \multicolumn{5}{|c|}{ Renda familiar per capita mensal: $(\mathrm{R} \$)^{* *}$} \\
\hline Quartil $1(<331,477)$ & 92 & 30,1 & 44 & 18,4 \\
\hline Quartil $2(331,488-500,00)$ & 79 & 25,8 & 76 & 31,8 \\
\hline Quartil $3(500,001-750,00)$ & 71 & 23,2 & 58 & 24,3 \\
\hline Quartil $4(>750,00)$ & 64 & 20,9 & 61 & 25,5 \\
\hline \multicolumn{5}{|l|}{ Escolaridade $(\%)^{* * *}$} \\
\hline Até 20 grau completo & 51 & 14,2 & 37 & 12,1 \\
\hline Superior incompleto & 138 & 38,5 & 59 & 20,4 \\
\hline Superior completo & 169 & 47,2 & 193 & 66,8 \\
\hline
\end{tabular}

das faixas de idade. Evidenciou-se tendência de aumento da mediana do peso com o avanço da idade em ambos os sexos, ainda que, para as mulheres, este comportamento tenha ocorrido somente até a faixa de 35-39 anos, estabilizando-se a seguir. A média do peso também foi maior entre homens $(78,0 \mathrm{~kg})$ do que entre muIheres $(61,6 \mathrm{~kg})(p=0,001)$, com tendência semelhante à da mediana.

As medianas da estatura para homens e mulheres foram $176,0 \mathrm{~cm}$ e $163,0 \mathrm{~cm}$, respectivamente. Observou-se aumento da mediana da estatura em mulheres até o grupo de 20 a 34 anos, e diminuição nas faixas subsequentes, que se acentuou a partir dos 45 anos. Para os homens, a mediana diminuiu até 34 anos, mantendo-se relativamente estável nas faixas etárias seguintes.

O IMC dos 605 funcionários com informação de peso e estatura mostrou-se substancialmente diferente entre os sexos. Sua média foi de $25,1 \mathrm{~kg} / \mathrm{m}^{2}$, para homens, e $23,3 \mathrm{~kg} / \mathrm{m}^{2}$, para mulheres $(p<0,001)$, enquanto a mediana do IMC foi de $24,5 \mathrm{~kg} / \mathrm{m}^{2}$ e $22,5 \mathrm{~kg} / \mathrm{m}^{2}$ para homens e mulheres, respectivamente.

Categorizando-se o IMC segundo a recomendação de 1995 (OMS), os homens apresen- taram freqüência maior de sobrepeso grau I $(36,0 \%$; IC $=31,5-40,5)$ do que as mulheres (17,0\%; IC =13,1-20,9) (Figura 1). Nas categorias baixo peso (IMC <18,5) e sobrepeso graus II e III, as proporções são substancialmente menores, e poucos indivíduos foram classificados nestas categorias. Assim, nas análises subseqüentes, a classificação de 1986 da OMS (OMS, 1986) será adotada, já que possibilita comparações com outros estudos e evita a rarefação dos dados.

A proporção de indivíduos com excesso de peso aumentou com a idade (Tabela 2), sendo maior entre os homens, em todas as faixas etárias. Os homens de 40 anos e mais apresentaram freqüência 2,1 vezes maior de sobrepeso/obesidade do que os da faixa de 20 a 34 anos. Já para as mulheres, a proporção de obesas na faixa etária de 40 anos e mais foi duas vezes maior do que nas mulheres com idade abaixo de 34 anos.

Os casados mostraram freqüência mais elevada de sobrepeso (31,0\%; IC =26,6-35,4) comparados aos solteiros $(20,5 \%$; IC $=14,5$ $26,5)$, contudo, em relação aos divorciados/ separados/ viúvos, não houve diferença significativa (25,6\%; IC =22,2-29,0) (Tabela 3). Embora nesta última categoria a proporção de sobrepeso tenha sido maior do que na dos solteiros, diferenças nas distribuições etárias parecem explicar melhor a associação. Assim, a idade foi variável de confundimento para a associação I M C/ situação conjugal, comparando-se divorciados/ separados/ viúvos aos solteiros $\left(\mathrm{OR}_{\mathrm{bruta}}=0,9 ; \mathrm{OR}_{\mathrm{MH}}=1,1\right)$.

As proporções de excesso de peso variaram segundo quartis de renda sem configurar, no entanto, padrão nítido de influência da renda sobre a massa corporal (Tabela 3). Entre os indivíduos com escolaridade até o 2 o grau, evidenciou-se a freqüência mais elevada de baixo peso $(17,7 \%$; IC $=15,1-20,4)$, comparada à daqueles com nível superior incompleto (7,7\%; IC $=5,5-9,2)$ e superior completo $(7,3 \%$; IC = $5,5-9,1)$ (Tabela 3). Todavia, como os funcionários com menor nível de escolaridade eram mais jovens do que aqueles com maior instrução, não se pode atribuir somente à menor escolaridade aquela freqüência. Não houve diferença entre as proporções de sobrepeso nos funcionários com até segundo grau completo $(24,1 \%$; IC $=21,1-27,1)$, superior incompleto $(25,1 \%$; IC $=22,1-28,1)$ e superior completo (30,0\%; IC =26,82-33,18) (Tabela 3).

Para proceder à comparação da freqüência de sobrepeso (IMC $\geq 25 \mathrm{~kg} / \mathrm{m}^{2}$ ) estimada entre os funcionários do Banco do Brasil (BB) com aquela estimada na população adulta, investi- 
gada na PNSN / 1989, para a região Sudeste urbana, foi necessário reagrupar os dados a fim de adequar a estratificação etária. Dessa forma, observa-se que para os homens estudados na PNSN e também no Banco, a proporção de IMC $\geq 25 \mathrm{~kg} / \mathrm{m}^{2}$ aumentou com a idade, encontrando-se freqüências mais elevadas em todas as faixas etárias nos bancários (Tabela 4). Para as mulheres, também houve incremento das proporções do IMC $\geq 25 \mathrm{~kg} / \mathrm{m}^{2}$ com o aumento da idade. No entanto, ao contrário do que foi observado entre os homens, na PNSN foram estimadas freqüências aproxi madamente duas vezes mais elevadas de sobrepeso/ obesidade nas faixas de 35 a 44 anos e 45 a 64 anos. Verifica-se ainda na tabela 4 que, enquanto na PNSN, as mulheres apresentaram prevalências mais elevadas de sobrepeso/obesi dade do que os homens, em todas as faixas de idade, no Banco do Brasil esta situação se inverteu.

No modelo de regressão múltipla linear (Tabela 5), o conjunto de variáveis que melhor explicaram a variação do IMC foram sexo, idade e escolaridade, sendo excluídas situação conjugal e renda familiar. Os coeficientes de regressão do modelo representam a variação em logaritmo do IMC (variável dependente), para cada unidade da variável independente. Assim, para "sexo" por exemplo, pode-se dizer que a diferença do logaritmo do IMC entre mulheres e homens foi em média -0,073 ( $\log$ IMC), o que significa maiores valores médios do IMC para os homens.

\section{Discussão}

Houve grande adesão e cooperação entre os funcionários do Banco do Brasil para a realização deste estudo. Isso pode ser atribuído ao alto nível de escolaridade e também, segundo Griep (1996), ao fato de o questionário ter sido respondido com garantia de sigilo das informações, durante o turno de trabalho, e em horários que não prejudicaram o desenvolvimento de suas funções.

Peso e estatura informados pelos próprios funcionários constituíram os dados principais deste estudo. Sua utilização foi possível pela validade que apresentaram na análise comparativa com a medida direta de uma sub-amostra de 321 funcionários ("padrão-ouro"), realizada por Chor (1997). De acordo com este "padrão-ouro", a sensibilidade da informação, categorizada em baixo peso/normal e sobrepeso/ obesidade, variou entre $76,0 \%$ e $96,0 \%$ nas quatro dependências do banco onde as medidas foram tomadas. A especificidade, por outro
Figura 1

Freqüência (\%) do IMC por sexo, em funcionários do Banco do Brasil - 1994/RJ .

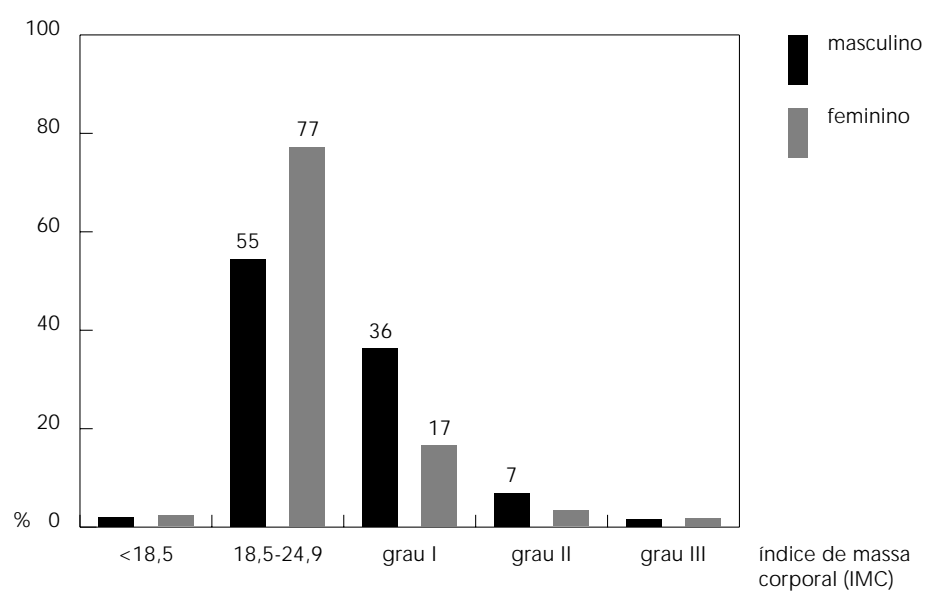

Tabela 2

Distribuição de freqüência de IMC elevado, segundo sexo e idade em funcionários do Banco do Brasil - 1994/RJ .

\begin{tabular}{lccccc}
\hline \multirow{2}{*}{ Idade (anos) } & \multicolumn{5}{c}{$\left(\mathrm{IMC} \geq 25 \mathrm{~kg} / \mathrm{m}^{2}\right)$} \\
& \multicolumn{2}{c}{ Masculino } & \multicolumn{2}{c}{ Feminino } & $\mathrm{p}$ \\
& $\mathrm{n}$ & $\%$ & $\mathrm{n}$ & $\%$ & \\
\hline $20-34$ & 42 & 34,1 & 07 & 15,2 & 0,001 \\
$35-39$ & 41 & 47,1 & 18 & 17,6 & 0,001 \\
$40 \mathrm{e}+$ & 68 & 68,7 & 31 & 31,3 & 0,001 \\
\hline
\end{tabular}

$\chi 2$ linear - homens $=8,0 ; p<0,001$

$\chi 2$ linear - mulheres $=3,5 ; p=0,06$

lado, situou-se próxima a 100,0\% em todas as dependências do Banco (Chor, 1997). Outros estudos (Palta et al., 1982; Schmidt et al., 1993) também evidenciaram a validade da informação do peso e estatura, tanto no Brasil, como em outros países.

Os bancários constituíram uma população relativamente jovem, na sua maioria casados, de perfil funcional semelhante, média salarial maior para homens, mas renda familiar per capita homogênea entre ambos os sexos. Os níveis de renda familiar per capita e de escolaridade indicam tratar-se de grupo que pertence, predominantemente, a um estrato social acima da média do país e do município.

Estudos realizados no Brasil e em outros países em desenvolvimento mostraram que as mulheres têm maior probabilidade de apresentar excesso de peso do que os homens (Coitinho et al., 1991; Lolio \& Latorre, 1991; Sichieri et al., 1994; Monteiro et al., 1995b). No en- 
Índice de Massa Corporal (IMC) segundo variáveis sócio-demográficas de funcionários do Banco do Brasil - 1994/RJ .

\begin{tabular}{|c|c|c|c|c|c|c|c|c|}
\hline & \multicolumn{8}{|c|}{ IMC } \\
\hline & \multicolumn{2}{|c|}{$\begin{array}{c}<20 \\
(\text { Baixo peso) }\end{array}$} & \multicolumn{2}{|c|}{$\begin{array}{c}20-24 \\
\text { (Normal) }\end{array}$} & \multicolumn{2}{|c|}{$\begin{array}{c}25-29 \\
\text { (Sobrepeso) }\end{array}$} & \multicolumn{2}{|c|}{$\begin{array}{c}\geq 30 \\
(\text { O besidade })\end{array}$} \\
\hline & $n$ & $\%$ & $n$ & $\%$ & $\mathrm{n}$ & $\%$ & $\mathrm{n}$ & $\%$ \\
\hline \multicolumn{9}{|l|}{ Situação conjugal* } \\
\hline Solteiros & 21 & 14,4 & 83 & 56,8 & 30 & 20,5 & 12 & 8,2 \\
\hline Casados & 25 & 6,6 & 215 & 28,4 & 118 & 31,0 & 23 & 6,0 \\
\hline Divorciados/Separados/Viúvos & 7 & 9,0 & 47 & 60,3 & 20 & 25,6 & 4 & 5,1 \\
\hline \multicolumn{9}{|c|}{ Renda familiar per capita mensal: $(\mathrm{R} \$)^{* *}$} \\
\hline Quartil $1(<331,48)$ & 14 & 10,7 & 63 & 48,1 & 45 & 34,4 & 9 & 6,9 \\
\hline Q uartil $2(331,48-500,00)$ & 9 & 6,3 & 93 & 64,6 & 31 & 21,5 & 11 & 7,6 \\
\hline Quartil $3(500,01-750,00)$ & 11 & 8,9 & 63 & 50,8 & 42 & 33,9 & 8 & 6,5 \\
\hline Quartil $4(>750,00)$ & 8 & 6,8 & 70 & 59,8 & 34 & 29,1 & 05 & 4,3 \\
\hline \multicolumn{9}{|l|}{ Escolaridade } \\
\hline A té 2 o grau completo & 14 & 17,7 & 46 & 58,2 & 19 & 24,1 & 00 & 0,0 \\
\hline Superior incompleto & 14 & 7,7 & 107 & 58,5 & 46 & 25,1 & 16 & 8,7 \\
\hline Superior completo & 25 & 7,3 & 192 & 56,0 & 103 & 30,0 & 23 & 6,7 \\
\hline
\end{tabular}

$* \chi 2=12,84, p=0,05$. Excluídos os sem informação

$* * \chi 2=12,44, p=0,19$. Excluídos os sem informação

tanto, verifica-se um incremento de sobrepeso/ obesidade com o aumento da renda para homens, mas não para mulheres, a partir do terceiro quartil de renda (Anjos, 1993; Sichieri et al., 1994). No Banco do Brasil, as funcionárias apresentaram proporções de sobrepeso/ obesidade muito menores do que os homens. Este fato pode estar refletindo 1) a maior preocupação do sexo feminino com a estética corporal, tendo em vista que nas sociedades modernas o padrão de elegância e beleza passa pela magreza (Anjos, 1993; Silva, 1995), e 2) diferenças entre homens e mulheres com relação a fatores comportamentais, como padrão de consumo alimentar, prática de atividades físicas, consumo de bebidas alcoólicas entre outros.

Quanto à escolaridade, estimou-se maior proporção de baixo peso entre os bancários com até segundo grau completo, enquanto proporções semel hantes de sobrepeso foram encontradas nos três níveis de escolaridade. Alguns estudos em países desenvolvidos evidenciaram forte correlação negativa entre escolaridade e excesso de peso, enquanto em países em desenvolvimento, incluindo o Brasil, estudos demonstraram associação positiva (Sobal, 1991; OMS, 1995). Entre os bancários, não foram encontradas diferenças importantes de prevalência de sobrepeso entre os níveis de escolaridade, provavelmente pela grande ho- mogeneidade deste grupo em relação ao nível de instrução e também pelo pequeno número de funcionários sem nível superior.

Na regressão linear, as variáveis sexo, idade e escolaridade (nível superior completo e incompleto) mantiveram-se significativas no modelo final. O sexo foi a variável mais importante (maior coeficiente de regressão padronizado), o que reforça os achados da análise estratificada para estas variáveis. A associação verificada entre situação conjugal e IMC na análise bivariada não se mostrou significativa quando ajustada pelas demais variáveis na regressão múltipla linear.

Para fins comparativos, a população da PNSN constituiu a melhor aproximação disponível da população em geral a ser tomada como referência para análise dos dados da população bancária. Dessa forma, os bancários tiveram medianas de peso e estatura mais elevadas do que as encontradas por Anjos (1993) em dados da PNSN, para a população urbana brasileira. Em relação ao peso, a diferença dos valores medianos entre os dois estudos foi de 9,83 kg para homens e 1,74 kg para mulheres. Em relação à estatura, as diferenças foram de $7,31 \mathrm{~cm}$ e 7,41 cm para homens e mulheres, respectivamente. Estas discrepâncias podem ser justificadas pela inclusão dos idosos somente na PNSN, que apresentam diminuição da estatura em relação à população economicamente ativa. 
Entre os bancários, assim como na PNSN (Anjos, 1993), valores de peso e estatura sistematicamente maiores foram estimados para os homens em todas faixas etárias. Quanto à estatura, na PNSN, houve diminuição das medianas com o decorrer da idade tanto em homens como em mulheres (Anjos, 1993). Já entre os bancários, a tendência de declínio foi observada de forma contundente para mulheres após os 30 anos de idade, enquanto, entre os homens, houve apenas uma leve propensão à queda, com oscilações entre as faixas. Tal diminuição pode ser explicada por fatores biológicos, sociais e ambientais, que ocorrem de forma diferenciada ao longo da vida das diversas coortes de idade. Nesse sentido, a perda na estatura, principalmente nas idades mais avançadas, deve-se ao processo natural de envelhecimento, à osteoporose, que se manifesta de forma mais acentuada em mulheres, e a problemas articulares, entre outros (Wardlaw, 1996). Tais fatores, entretanto, não são suficientes para explicar a redução da estatura observada no grupo de bancários com idade acima de 30 anos, considerando-se que esta é uma população relativamente jovem. Tratando-se de estudo seccional, em que várias coortes de idade estão representadas, é plausível que a diferença observada seja explicada pela tendência de elevação da estatura nas gerações mais jovens (Monteiro et al., 1995a).

Quando comparamos as freqüências do IMC da PNSN para a região Sudeste com as apresentadas pelos bancários, percebemos que o percentual de indivíduos com IMC igual ou maior do que $25 \mathrm{~kg} / \mathrm{m}^{2}$ é elevado tanto na PNSN, como nos bancários, em ambos os sexos. Todavia, enquanto os homens do Banco do Brasil apresentaram freqüências de sobrepeso/ obesidade maiores do que os da PNSN, as mulheres bancárias tiveram proporções menores, em todas as faixas de idade. Estudos em populações de países desenvolvidos mostram que o nível sócio-econômico está inversamente associado à obesidade nas mulheres adultas (Sobal, 1991). Assim, os resultados da análise comparativa entre os dois estudos sugerem que o perfil antropométrico, principalmente das mulheres bancárias, aproxima-se do perfil de populações mais favorecidas economicamente e não segue o padrão da população em geral.

As diferenças evidenciadas entre o perfil antropométrico dos dois estudos, em relação ao sexo, retratam e são resultantes de aspectos distintos que envolvem a população da região Sudeste e grupos populacionais específicos que a compõem, como os bancários. O fator que pode
Tabela 4

Distribuição percentual de categorias do índice de massa corporal (IMC), por sexo e idade, da população urbana da região Sudeste (Pesquisa Nacional Sobre Saúde e Nutrição/PNSN*-1989) e dos funcionários do Banco do Brasil** (BB) - 1994/RJ .

\begin{tabular}{|c|c|c|c|c|}
\hline \multirow[t]{2}{*}{ Sexo e idade } & \multirow{2}{*}{$\begin{array}{c}n \\
\text { PNSN }\end{array}$} & \multirow{2}{*}{$\begin{array}{c}n \\
\text { B.B. }\end{array}$} & \multicolumn{2}{|c|}{$I M C \geq 25$} \\
\hline & & & $\%$ & $\%$ \\
\hline \multicolumn{5}{|l|}{ Homens } \\
\hline $18-34$ & 7.713 .431 & 123 & 22,8 & 34,1 \\
\hline $35-44$ & 3.511 .261 & 184 & 39,1 & 48,9 \\
\hline $45-64$ & 4.019 .628 & 34 & 42,6 & 55,9 \\
\hline \multicolumn{5}{|l|}{ Mulheres } \\
\hline $18-34$ & 7.956 .822 & 46 & 27,4 & 15,2 \\
\hline $35-44$ & 3.504 .172 & 196 & 48,8 & 21,4 \\
\hline $45-64$ & 4.504 .897 & 22 & 60,2 & 31,8 \\
\hline
\end{tabular}

* Fonte: COITINHO et al., 1991

** Faixa etária entre 20 a 60 anos.

Tabela 5

Coeficientes, intervalo de confiança (IC) e p-valor do modelo de regressão linear. Variável dependente: IMC, em funcionários do Banco do Brasil - 1994/RJ.

\begin{tabular}{|c|c|c|c|c|}
\hline & $\beta_{\text {bruto }}$ & $\beta_{\text {ajustado }}$ & IC & $\mathrm{p}$ \\
\hline \multicolumn{5}{|l|}{ Sexo } \\
\hline Homens & - & - & - & - \\
\hline Mulheres & $-0,073$ & $-0,083$ & $-0,110-0,062$ & 0,001 \\
\hline Idade (variável contínua) & 0,003 & 0,005 & $0,003-0,006$ & 0,001 \\
\hline \multicolumn{5}{|l|}{ Escolaridade } \\
\hline Até 20 grau completo & - & - & - & - \\
\hline Superior incompleto & 0,0422 & 0,038 & $3,7141 \mathrm{E}-05-0,076$ & 0,050 \\
\hline Superior completo & 0,0493 & 0,052 & $0,0169-0,087$ & 0,004 \\
\hline
\end{tabular}

ser apontado de forma mais imediata para explicar estas diferenças é a situação econômicosocial, considerando-se que, quanto ao poder aquisitivo, à escolaridade e acesso às informações, os bancários constituíram um grupo privilegiado, quando comparados à população em geral.

As proporções de sobrepeso definidas através do IMC em bancários foram elevadas principalmente em homens, e o excesso de peso neste grupo é preocupante, na medida em que representa um sobre-risco de morbidade, invalidez e mortalidade, com enorme custo social. É bom lembrar que a principal causa de morte entre os funcionários do sexo masculino, para o período entre 1977 e 1990, foram as doenças 
cardiovasculares, com 38\% do total de óbitos (Beltrão et al., 1996). Por outro lado, a compreensão dos elementos culturais e comportamentais que contribuíram para a menor prevalência de sobrepeso entre as bancárias poderia auxiliar no desenvolvimento de estratégias de prevenção e controle.
Uma vez que essa categoria profissional assemel ha-se em termos de condições de trabaIho e de vida a um segmento expressivo da população, os resultados aqui apresentados interessam diretamente ao Banco do Brasil, mas também à sociedade em geral, já que apontam a necessidade de definir e desenvolver medidas de prevenção e controle da obesidade queé, cada vez mais, um problema de saúde pública.

\section{Referências}

ANJOS, L. A., 1993. Valores Antropométri cos da População Adulta Brasileira: Resultados da Pesquisa Nacional sobre Saúdee Nutrição. Tese apresentada ao Departamento de Nutrição Social da Universidade Federal Fluminense para concorrer à vaga de professor titular em Nutrição e Saúde Pública. Rio de Janeiro: Universidade Federal Fluminense.

ASHTON - TATE, 1988. Manual de Referência - dBASE IV. São Paulo: Datalógica.

BELTRÃO, I. K.; DUCHIADE, M. P.; CHOR, D.; VALENTE, J.; FONSECA, M. M. J.; ANDRADE, C. R. \& LIMA, J. M., 1996. Pesquisa de Saúde dos Associados da CASSI - relatório final, Vol. I e II. Rio de Janeiro: Ence-FIBGE/Ensp-Fiocruz.

BLOCH, K. V.; KLEIN, C. H.; SILVA, N. A. S.; NOGUEIRA, A. R. \& CAM POS, L. H. S., 1994. Hipertensão arterial e obesidade na Il ha do Governador, Rio de Janeiro. Arquivos Brasileiros de Cardiologia, 62:17-22.

COITINHO, D. C.; LEÃO, M. M.; RECINE, E. \& SICHIERI, R., 1991. Condições Nutricionais da População Brasileira: Adultos e Idosos - Pesquisa Nacional Sobre Saúde e Nutrição. Brasília: INAN (Instituto Nacional de Alimentação e Nutrição).

CHOR, D., 1997. Perfil de Risco Cardiovascular de Funcionários de Banco Estatal. Tese de Doutorado, São Paulo: Faculdade de Saúde Pública, Universidade de São Paulo.

DUNCAN, B. B.; SCHMIDT, M. I.; POLANCZYK, C. A.; HOMRICH, C. S.; ROSA, R. S. \& ACHUTTI, A. C., 1993. Fatores de risco para doenças não-transmissíveis em área metropolitana na região sul do Brasil: prevalência e simultaneidade. Revista de Saúde Pública, 27:143-8.

GIBSON, R. S., 1990. Principles of Nutritional Assessment. Oxford: Oxford University Press.

GRIEP, R. H., 1996. Tabagismo entre Trabalhadores de um Banco Estatal. Dissertação de Mestrado, Rio de Janeiro: Escola Nacional de Saúde Pública, Fundação Oswaldo Cruz.
KAHN, H. A. \& SEMPOS, C. T., 1989. Statistical Methods in Epidemiology. New York/Oxford: Oxford University Press.

LESSA, I.; MENDONÇA, G. A. S. \& TEIXEIRA, M. T. B., 1996. Doenças crônicas não transmissíveis no Brasil: dos fatores de risco ao impacto social. Boletín dela Oficina Sanitaria Panamericana, 120: 389-413.

LOLIO, C. A. \& LATORRE, M. R. D. O., 1991. Prevalência de obesidade em localidade do Estado de São Paulo, Brasil, 1987. Revista de Saúde Pública, 23: 33-36.

MONTEIRO, C. A.; BENÍCIO, M. H. D’A. \& GOUVEIA, N. C., 1995a. Evolução da altura dos brasileiros. In: Vel hos e Novos Males da Saúde no Brasil - A Evolução do País e de suas Doenças (C. A. Monteiro, org.), pp. 126-140, São Paulo: Hucitec-Nupens/USP.

MONTEIRO, C. A.; MONDINI, L.; SOUZA, A. L. M. \& POPKIN, B. M., 1995b. Da desnutrição para a obesidade: a transição nutricional no Brasil. In: Vel hos e Novos Males da Saúde no Brasil - A EvoIução do País e de suas Doenças (C. A. Monteiro, org.), pp. 247-255, São Paulo: Hucitec-Nupens/ USP.

NORUSIS, M. S., 1992. SPSS for Windows: Base System User's Guide Rel ease 5.0. Chicago.

OMS (Organização Mundial da Saúde), 1986. Use and interpretation of anthropometric indicators of nutritional status. Bulletin of the World Health Organization, 64:929-941.

OMS (Organização Mundial da Saúde), 1990. Dieta, Nutrición y Prevención de Enfermedades Crónicas. (Serie de Informes Técnicos, 797). Genebra: OMS.

OMS (Organização Mundial da Saúde), 1995. Physical Status: The Useand Interpretation of Anthropometry. (Technical Report Series, 854). Genebra: OMS.

PALTA, M.; PRINEAS, R. J.; BERMAN, R. \& HANNAN, P., 1982. Comparison of self-reported and measured height and weight. American Journal of Epidemiology, 115:223-230. 
POPKIN, B., 1994. The nutrition transition in low-income countries: an emerging crisis. Nutrition Reviews, 52:285-98.

PRATA, R. P., 1992. A transição epidemiológica no Brasil. Cadernos de Saúde Pública, 8:168-175.

REGO, R.; BERNARDO, F. A. N.; RODRIGUES, S. S. R.; OLIVEIRA, Z. M. A.; OLIVEIRA, M. B.; VASCONCELOS, C.; AVENTURADO, L. V. O.; MONCAU, J. E. C. \& RAM OS, L. R., 1990. Fatores de risco para doenças crônicas não-transmissíveis: inquérito domiciliar no município de São Paulo, SP (Brasil). Metodologia e resultados preliminares. Revista de SaúdePública, 24:277-85.

RIPPE, J. M., 1996. Overweight and health: communications challenges and opportunities. American Journal of Clinical Nutrition, 63:470S-473S.

ROU QUAYROL, M. Z.; VERAS, F. M. F.; VASCONCELOS, J. S.; BEZERRA, R. C. F.; GOMES, I. L. P. \& BEZERRA, F. A. F., 1987. Fatores de risco na doença coronariana: inquérito epidemiológico em estratos habitacionais de um bairro de Fortaleza. Arquivos Brasileiros de Cardiologia, 46: 339-347.

SICHIERI, R.; COITINHO, D. C.; LEÃO, M. M.; RECINE, E. \& EVERHART, J. E., 1994. High temporal, geographic, and income variation in body mass index among adults in Brazil. American Journal of Public Health, 84:793-798.

SCHMIDT, M. I.; DUNCAN, B. B.; TAVARES, M.; POLANCZYK, C. A.; PELLANDA, L. \& ZIMMER, P. M., 1993. Validity of self-reported weight - a study of urban brazilian adults. Revista de Saúde Pública, 27:271-276.

SILVA, D. O., 1995. O Fiel da Balança na História do Corpo Obeso de Mulheres de Baixa Renda - Manguinhos, Rio de Janeiro. Dissertação de Mestrado, Rio de Janeiro: Escola Nacional de Saúde Pública, Fundação Oswaldo Cruz.

SOBAL, J., 1991. Obesity and socioeconomic status: a framework for examining relationships between physical and social variables. Medical Anthropology, 13:231-247.

WARDLAW, G. M., 1996. Putting body weight and osteoporosis into perspective. American Journal of Clinical Nutrition, 63:433S-436S.

WOLF, A. M. \& COLDITZ, G. A., 1996. Social and economic effects of body weight in the United States. American Journal of Clinical Nutrition, 63:466S469S. 